\title{
Dimensionally Dependent Tensor Identities by Double Antisymmetrisation.
}

\author{
S. Brian Edgar* and A. Höglund ${ }^{\dagger}$ \\ Matematiska institutionen, Linköpings universitet, SE-581 83 Linköping, Sweden.
}

(Dated: November 4, 2018)

Some years ago, Lovelock showed that a number of apparently unrelated familiar tensor identities had a common structure, and could all be considered consequences in $n$-dimensional space of a pair of fundamental identities involving trace-free $(p, p)$-forms where $2 p \geq n$. We generalise Lovelock's results, and by using the fact that associated with any tensor in $n$-dimensional space there is associated a fundamental tensor identity obtained by antisymmetrising over $n+1$ indices, we establish a very general 'master' identity for all trace-free $(k, l)$-forms. We then show how various other special identities are direct and simple consequences of this master identity; in particular we give direct application to Maxwell, Lanczos, Ricci, Bel and Bel-Robinson tensors, and also demonstrate how relationships between scalar invariants of the Riemann tensor can be investigated in a systematic manner.

\section{INTRODUCTION}

In an $n$-dimensional space any tensor expression $T_{a_{1} a_{2} \ldots a_{k}}$ with $k>n$ indices satisfies a tensor identity

$$
T_{\left[a_{1} a_{2} \ldots a_{k}\right]}=0 .
$$

Such mathematically obvious identities can be very useful in practical calculations. However, the antisymmetrisation process may not be so explicit since it need not be applied only on free indices; it could also involve dummy indices, some of which could be absorbed into traces, as we shall demonstrate in the following example. (Here, and in the rest of this paper, we use the abstract index notation [21]. However, any index notation would probably work well as long as the above property holds.)

Example I.1. Dianyan Xu [6] constructed the following two scalar identities for the Riemann tensor in four dimensional spaces,

$$
R_{b}^{a} R^{b c d e} R_{a c d e}=\frac{1}{4} R R^{a b c d} R_{a b c d}+2 R^{a c} R^{b d} R_{a b c d}+2 R^{a}{ }_{b} R_{c}^{b} R_{a}^{c}-2 R R_{b}^{a} R_{a}^{b}+\frac{1}{4} R^{3}
$$

and

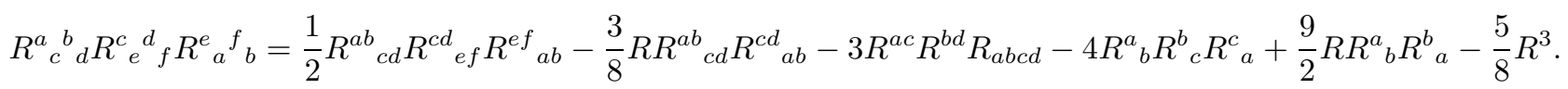

These were obtained after a lengthy calculation by decomposing the Riemann tensor $R_{a b c d}$ into its Weyl and Ricci components, and then using spinor methods; in fact Dianyan Xu claimed that it was not possible to obtain these identities from the algebraic properties of the Riemann tensor alone.

However, it was pointed out by Harvey [14] that the first identity could be obtained directly by expanding

$$
R_{[a b}^{a b} R_{c d}^{c d} R_{e]}^{e}=0
$$

and the second identity by expanding

$$
R_{[a b}^{a b} R_{c d}^{c d} R_{e f]}^{e f}=0
$$

and combining the result with the first identity.

So it is now clear that such identities exist not only for Riemann tensors, nor even just for Riemann candidates (any 4-tensors with the index symmetries of a Riemann tensor); they are in fact valid for any tensors which permit the antisymmetrisation constructions (4) and (5).

*Electronic address: bredg@mai.liu.se

${ }^{\dagger}$ Electronic address: anhog@mai.liu.se 
Harvey's approach also confirmed that both identities were valid in four and lower dimensions - irrespective of the signature of the space (a fact that was missing from Dianyan Xu's spinor derivation). So this insight into the structure of the two identities not only led to a better appreciation of the relevance of these identities in the original context (concerning counterterms in Lagrangians [14, 15, 16]), but also highlights the importance of identities built from the antisymmetrisation property (11) in the study of Riemann scalar invariants.

Of course the pattern and discussion above suggest how to obtain many more analogous identities for other tensors, for higher dimensions, and for higher order. In fact the identity (3) can be obtained more directly and compactly by instead using the Weyl tensor $C_{a b c d}$ (the trace-free part of the Riemann tensor) in the identity $C^{a b}{ }_{[a b} C^{c d}{ }_{c d} C^{e f}{ }_{e f]}=$ 0 [11, 14, 16].

It is also interesting to note that it is not just scalar identities which can be constructed in this manner; for example, similar tensor identities can be considered to underlie the familiar Cayley-Hamilton theorem:

Example I.2. Antisymmetrising over $n+1$ indices for matrices $M^{a}{ }_{b}$ in $n$ dimensions gives the Cayley-Hamilton theorem

$$
M^{c_{1}}{ }_{\left[c_{1}\right.} M^{c_{2}}{ }_{c_{2}} \ldots M^{c_{n}}{ }_{c_{n}} \delta_{a]}^{b}=0 .
$$

Considering the Cayley-Hamilton theorem from this viewpoint suggests generalisations involving more than one matrix,

$$
M^{c_{1}}{ }_{\left[{ }_{1}\right.} N^{c_{2}}{ }_{c_{2}} \ldots P^{c_{n}}{ }_{c_{n}} \delta_{a]}^{b}=0
$$

This generalised Cayley-Hamilton theorem has been used to find relations, (syzygies), between scalar invariants of matrices involving more than one matrix [20, 26, 27].

An important question is whether this technique of antisymmetrising is just a useful "trick" in very special circumstances, or whether there is deeper structure to be better understood and more fully exploited.

Some time ago Lovelock 19 noted the significance of certain types of identities - which he called dimensionally dependent identities - and demonstrated their existence and importance in quite a wide context (Lovelock [19] has defined a dimensionally dependent identity as an identity which is a direct consequence of the dimension of the space taking on a particular value, and which therefore is not valid for arbitrary dimension $n$ in general). By showing precisely how and where dimensionality plays its role in familiar identities, Lovelock revealed a technique which could be generalised to arbitrary dimension. In fact Lovelock's technique simply involved the antisymmetrising process being applied in $n$ dimensions, to tensors with two sets of indices (upper and lower, in practice) and specialised in two theorems to trace-free tensors with an equal number $p$ of upper and lower indices where $2 p \geq n$.

Lovelock's investigations were motivated mostly by familiar four dimensional identities satisfied by Weyl tensors (and Weyl candidates, i.e. tensors with the algebraic symmetries of the Weyl tensor); however, there are other identities which appear to be of a similar nature, but which cannot be confirmed by Lovelock's two theorems. It is the purpose of this paper to investigate dimensionally dependent identities in a systematic manner and obtain a more complete picture; in doing so we develop results more general than Lovelock's, and demonstrate that these new results can be used to confirm identities which cannot be obtained from Lovelock's results, and also to confirm other identities which can only be confirmed indirectly from Lovelock's results.

In the next section we make some general observations and present some examples as further motivation for the subsequent sections. We summarise and illustrate Lovelock's results [19] in Section [II. Lovelock's theorems applied only to trace-free $(p, p)$-forms in $n$ dimensions where $2 p \geq n$. However, in Section IV, we shall develop more general results in the form of a "master" identity which will be applicable to any tensor in any dimension, and in particular to trace-free $(k, l)$-forms in any dimension. In Section 5 we will then show that a number of important identities are consequences of this master identity. In particular, we show that the simplification of the gravity-matter coupling terms in the Weyl wave equation and in the Bel tensor, and the complete symmetry of the super-energy tensor for the Lanczos potential of the Weyl tensor are all trivial consequences when the master identity is specialised to four dimensions; in a similar manner we confirm the complete symmetry of the Bel-Robinson tensor in four and five dimensions. In addition, we demonstrate how the results permit a systematic study of relationships between scalar invariants of the Riemann tensor. In section 6 we illustrate how the importance of dimensionally dependent identities have been overlooked by reducing the algebraic Rainich condition, and point out the potential usefullness of these results in more general situations. 


\section{IDENTITIES BY DOUBLE ANTISYMMETRISATION.}

We consider the tensor $T^{\mathcal{A}}{ }_{a_{1}}$ where we have adopted the convention that $\mathcal{A}$ denotes an arbitrary number of additional lower and/or upper indices [21].

Associated with this tensor $T^{\mathcal{A}}{ }_{a_{1}}$, in $n$-dimensional space, there will always be an identity

$$
T^{\mathcal{A}}{ }_{\left[a_{1}\right.} \delta_{a_{2}}^{b_{2}} \delta_{a_{3}}^{b_{3}} \ldots \delta_{\left.a_{n+1}\right]}^{b_{n+1}}=0 .
$$

For future reference we should note that such identities cannot be made 'simpler', in the sense that taking the trace of (8) (on explicit indices, i.e., indices which are not implicit in $\mathcal{A}$ ) simply gives zero on the left hand side also.

An alternative approach would be to assume the presence of a volume element $\eta_{a_{1} a_{2} \ldots a_{n}}$, and formulate analogous results in the $\eta$ notation making use of duals; but we shall concentrate on developing results using the $\delta$ notation.

There will of course be one such identity associated with each separate index on $T$. We could obtain other identities, with less deltas, by taking more than one index of $T$ explicitly into the antisymmetrisation operation, but these new identities would involve only the antisymmetrised part of $T$ with respect to the explicit indices.

However, when the tensor $T$ is in fact antisymmetric in a group of $k$ indices, then we can get an identity involving less deltas than the original identity

$$
T^{\mathcal{A}}{ }_{\left[a_{1} \ldots a_{k}\right.} \delta_{a_{k+1}}^{b_{k+1}} \delta_{a_{k+2}}^{b_{k+2}} \ldots \delta_{\left.a_{n+1}\right]}^{b_{n+1}}=0
$$

but without losing any part of $T$. Again, it is important to note that taking the trace of (9), on explicit indices, simply gives zero on the left hand side also.

We next consider a tensor with both upper and lower indices, each of which contain groups of antisymmetric indices, i.e., $T^{\mathcal{A}}{ }_{a_{1} \ldots a_{k}}^{b_{1} \ldots b_{l}}=T^{\mathcal{A}}{ }_{\left[a_{1} \ldots a_{k}\right]}^{\left[b_{1} \ldots b_{l}\right]}$ is a $(\mathbf{k}, \mathbf{l})$-form with respect to its explicit indices; then we have the associated identity

$$
T^{\mathcal{A}}{ }_{a_{1} \ldots a_{k}}{ }^{\left[b_{1} \ldots b_{l}\right.} \delta_{a_{k+1}}^{b_{k+1}} \ldots \delta_{a_{n+1}}^{\left.b_{n+1}\right]}=0
$$

when $l \geq k$. (The analogous case obtained by antisymmetrising on the lower indices for $k \leq l$ is obvious.)

Such classes of tensors include many familiar tensors (Riemann, Weyl, Ricci, Maxwell, Lanczos, torsion) and they shall be the main focus of our investigation in this paper.

It is important to note that, although some traces of (10) yield the trivial identity, there are some traces of (10) which yield a non-trivial identity involving the trace of $T$. It is these non-trivial traces which yield Lovelock's identities, and our generalisations of them. We illustrate this with the following example.

Example II.1. We consider the $(2,2)$-form $R_{a b} c d$.

In three dimensions: $R_{e f}{ }^{\left[{ }^{h} h\right.} \delta_{a}^{c} \delta_{b}^{d]}=0$.

Contracting once over $e$ and $g$ gives,

$$
R_{f[a}{ }^{[h c} \delta_{b]}^{d]}-R_{e f}^{e[h} \delta_{a}^{c} \delta_{b}^{d]}=0,
$$

and then over $h$ and $f$ gives,

$$
R_{a b}{ }^{c d}-4 R_{e[a}{ }^{e[c} \delta_{b]}^{d]}+R_{e f}^{e f} \delta_{a}^{[c} \delta_{b}^{d]}=0 .
$$

From this last result follows the well-known fact that the trace-free part of a Riemann tensor is identically zero in three dimensions.

In four dimensions: $R_{g h}{ }^{[i j} \delta_{a}^{d} \delta_{b}^{e} \delta_{c}^{f]}=0$.

Contracting once over $g$ and $i$ gives,

$$
3 R_{h[a}^{[j d} \delta_{b}^{e} \delta_{c]}^{f]}-2 R_{g h}^{g[j} \delta_{a}^{d} \delta_{b}^{e} \delta_{c}^{f]}=0
$$

and then over $h$ and $j$ gives,

$$
3 R_{[a b}^{[d e} \delta_{c]}^{f]}-6 R_{g[a}^{g[d} \delta_{b}^{e} \delta_{c]}^{f]}+R_{g h}^{g h} \delta_{a}^{[d} \delta_{b}^{e} \delta_{c}^{f]}=0 .
$$

A third contraction gives zero on the left hand side also.

All of the above identities can be expressed in a more concise form if $R_{a b c d}$ is decomposed into trace-free and trace parts. 
We note that certain of these contractions yield identities which, if we were to encounter them not knowing their source, would seem (to our surprise) to come from antisymmetrising over $n$ or $n-1$ explicit indices in $n$ dimensions; on the otherhand, when one takes into account all the terms in each identity and also notes that these contractions involve antisymmetrising over upper and lower indices, of course, we would realise that our first judgement was superficial, and we have a disguised antisymmetrisation over $n+1$ indices. However, we have noted above that the identities exist in their most concise form when presented in terms of the trace free part of $R_{a b c d}$; so such identities can be even more deceptive especially when constructed explicitly in terms of a trace-free tensor.

So we now specialise to the important special situation where the $(k, l)$-form $T^{\mathcal{A}}{ }_{a_{1} \ldots a_{k}} b_{1} \ldots b_{l}=T^{\mathcal{A}}\left[a_{1} \ldots a_{k}\right]{ }^{\left[b_{1} \ldots b_{l}\right]}$ is trace-free, i.e., $T^{\mathcal{A}}{ }_{a_{1} a_{2} \ldots a_{k}}{ }^{a_{1} b_{2} \ldots b_{l}}=0$, and the underlying structure of the resulting identities become less transparent. To illustrate this we will give three simple examples involving respectively a trace-free $(2,2)$-form $W_{a b} c d$ (of which the Weyl conformal curvature tensor $C_{a b}{ }^{c d}$ is a special example being a symmetric trace-free $(2,2)$-form since $C_{a b c d}=$ $C_{c d a b}$, with the additional property $C_{[a b c] d}=0$ ), a trace-free $(2,1)$-form $L_{a b}{ }^{c}$ (of which the torsion and Lanczos potential with the additional property $L_{[a b c]}=0$ [17] are special examples), and a trace-free $(1,1)$-form $S^{a}{ }_{b}($ of which the trace-free symmetric Ricci tensor $\tilde{R}_{b}^{a}$, the trace-free symmetric energy tensor $\tilde{T}_{b}^{a}$ and the antisymmetric Maxwell tensor $F_{b}^{a}$, are special examples).

Example II.2. We now apply the above arguments to the trace-free $(2,2)$-form $W_{a b}{ }^{c d}$.

In three dimensions: $W_{e f}^{\left[{ }^{[g h}\right.} \delta_{a}^{c} \delta_{b}^{d]}=0$.

Contracting over $f$ and $h$ gives

$$
W_{e\left[a^{[g c}\right.} \delta_{b]}^{d]}=0,
$$

and then over $e$ and $g$ gives

$$
W_{a b}^{c d}=0
$$

In four dimensions: $W_{g h}{ }^{[i j} \delta_{a}^{d} \delta_{b}^{e} \delta_{c}^{f]}=0$.

Contracting over $h$ and $j$ gives

$$
W_{g\left[a^{[i d}\right.} \delta_{b}^{e} \delta_{c]}^{f]}=0,
$$

and then over $g$ and $i$ gives

$$
W_{[a b}^{[d e} \delta_{c]}^{f]}=0 .
$$

A third contraction gives zero on the left hand side also.

In five dimensions: $W_{i j}{ }^{[k l} \delta_{a}^{e} \delta_{b}^{f} \delta_{c}^{g} \delta_{d}^{h]}=0$.

Contracting over $j$ and $l$ gives

$$
W_{i\left[a^{[k e}\right.} \delta_{b}^{f} \delta_{c}^{g} \delta_{d]}^{h]}=0
$$

and then over $i$ and $k$ gives

$$
W_{[a b}^{[e f} \delta_{c}^{g} \delta_{d]}^{h]}=0 .
$$

A third contraction gives zero on the left hand side also.

So, in each of the three cases, the first identity is obvious in the sense that it is an explicit antisymmetrisation over $n+1$ indices in $n$ dimensions. What is particularly interesting, and at first sight perhaps surprising in these situations, is the existence of simple identities in $n$ dimensions which involve explicit antisymmetrization over only $n-1$ indices. But of course, in addition, there is antisymmetrisation on both lower and upper indices and the comparatively simple versions are due to the vanishing of the trace of $W$.

Example II.3. Consider the trace-free $(2,1)$-form $L_{a b}{ }^{c}$.

When we apply the above arguments we obtain,

In three dimensions: $L_{[f a}^{g} \delta_{b}^{d} \delta_{c]}^{e}=0$. 
Contracting once on the upper index on $L$ gives

$$
L_{[a b}^{[d} \delta_{c]}^{e]}=0,
$$

and contracting once more gives zero on the left hand side also.

In four dimensions: $L_{[h a}{ }^{i} \delta_{b}^{e} \delta_{c}^{f} \delta_{d]}^{g}=0$.

Contracting once on the upper index on $L$ gives

$$
L_{[a b}{ }^{[e} \delta_{c}^{f} \delta_{d]}^{g]}=0,
$$

and once more gives zero on the left hand side also.

So, once again, we obtain identities in $n$ dimensions involving explicit antisymmetrization over less than $n+1$ indices; although in this case it involves $n$ indices.

Example II.4. Consider the trace-free $(1,1)$-form $S_{a}{ }^{b}$.

When we apply the above arguments we obtain,

In three dimensions: $S_{[g}{ }^{h} \delta_{a}^{d} \delta_{b}^{e} \delta_{c]}^{f}=0$.

Contracting once on the upper index on $S$ gives

$$
S_{[a}^{[d} \delta_{b}^{e} \delta_{c]}^{f]}=0,
$$

and contracting once more gives zero on the left hand side also.

$\underline{\text { In four dimensions: }} S_{[i}{ }^{j} \delta_{a}^{e} \delta_{b}^{f} \delta_{c}^{g} \delta_{d]}^{h}=0$.

Contracting once on the upper index on $S$ gives

$$
S_{[a}^{[e} \delta_{b}^{f} \delta_{c}^{g} \delta_{d]}^{h]}=0,
$$

and once more gives zero on the left hand side also.

So, once again, we obtain identities in $n$ dimensions involving explicit antisymmetrization over only $n$ indices.

In the above examples we have obtained obvious identities by antisymmetrisation over $n+1$ indices in $n$ dimensions; but in addition, as a result of taking traces, we have obtained less obvious identities containing less deltas and explicit symmetrisation over less than $n+1$ indices. The existence of these additional identities, which contain less deltas than the obvious ones, are very important for building up more complicated identities. For example, consideration of the obvious four dimensional identity for the Weyl tensor $C_{[a b}{ }^{f g} \delta_{c}^{h} \delta_{d}^{i} \delta_{e]}^{j}=0$ (with 10 free indices), would not suggest the possibility of any third order scalar identities for $C$; on the otherhand, the less obvious one formed from the double trace, $C_{[a b}^{[f g} \delta_{c]}^{h]}=0$ (with 6 free indices), certainly permits the constructions of third order Weyl scalar identities by multiplication with a pair of $C$ tensors.

For each case considered we have gone as far as we could, in the sense that taking an additional trace simply reduces the left hand side to zero. Nevertheless, this does not in itself mean that there could not exist additional identities with even less deltas than those given above; however, for each of the cases it can be shown directly that removing further deltas gives a restriction rather than an identity, e.g., in four dimensions, when the trace is calculated for $L_{[a b}^{[c} \delta_{f]}^{g]}=0$ we find that $L_{a b}{ }^{c}=0$.

In the next section we shall give results due to Lovelock 19] and show that the results for three and four dimensions of Example II.2 follow directly as a special case; it will also be shown that the result for five dimensions can be deduced, more subtly, from Lovelock's results. In Section IV we will derive even more general results of which Lovelock's and the above three examples will be seen to be special cases.

\section{LOVELOCK'S DIMENSIONALLY DEPENDENT IDENTITIES}

Some years ago, Lovelock 19] noted that familiar tensors, (such as the Weyl and Maxwell tensors), with antisymmetry and trace-free properties, obeyed assorted - apparently unrelated — identities. However, although there was no common structural link in the original derivations of these assorted identities, Lovelock [19] showed that they could all be considered to be consequences of two underlying basic tensor identities. These underlying identities had a very simple structure and were a mathematically trivial consequence of dimension alone. We will now quote Lovelock's two theorems, each of which he proved in two different ways; our proofs are essentially conciser presentations of one of his versions. 
Theorem III.1. In an n-dimensional space let $T^{\mathcal{A}}{ }_{a_{1} \ldots a_{k}}{ }^{b_{1} \ldots b_{k}}=T^{\mathcal{A}}{ }_{\left[a_{1} \ldots a_{k}\right]}{ }^{\left[b_{1} \ldots b_{k}\right]}$ be trace-free on its explicit indices. If $2 k>n$ then

$$
T^{\mathcal{A}}{ }_{a_{1} \ldots a_{k}} b_{1} \ldots b_{k}=0
$$

Proof. Since $2 k>n$, antisymmetrising over $2 k$ indices gives an identity

$$
0=T_{\left[a_{1} \ldots a_{k}\right.}^{i_{1} \ldots i_{k}} \delta_{i_{1}}^{b_{1}} \ldots \delta_{\left.i_{k}\right]}^{b_{k}} .
$$

Since the tensor $T$ is trace-free, we get

$$
0=T^{\mathcal{A}}{ }_{a_{1} \ldots a_{k}}^{i_{1} \ldots i_{k}} \delta_{i_{1}}^{b_{1}} \ldots \delta_{i_{k}}^{b_{k}}
$$

Absorbing the deltas gives the theorem.

Theorem III.2. In an n-dimensional space let $T^{\mathcal{A}}{ }_{a_{1} \ldots a_{k}}{ }^{b_{1} \ldots b_{k}}=T^{\mathcal{A}}{ }_{\left[a_{1} \ldots a_{k}\right]}^{\left[b_{1} \ldots b_{k}\right]}$ be trace-free on its explicit indices. If $2 k=n$ then

$$
T^{\mathcal{A}}{ }_{\left[a_{1} \ldots a_{k}\right.}^{\left[b_{1} \ldots b_{k}\right.} \delta_{\left.a_{k+1}\right]}^{\left.b_{k+1}\right]}=0
$$

Proof. The proof is analogous to the proof of Theorem III.1 but this time the antisymmetrisation is over $2 k+1$ indices. Starting with

$$
0=T_{\left[a_{1} \ldots a_{k}\right.}^{{ }^{i_{1} \ldots i_{k}}} \delta_{a_{k+1}}^{b_{k+1}} \delta_{i_{1}}^{b_{1}} \ldots \delta_{\left.i_{k}\right]}^{b_{k}}=T^{\mathcal{A}}{ }_{\left[a_{1} \ldots a_{k}\right.}^{i_{1} \ldots i_{k}} \delta_{a_{k+1}}^{\left[b_{k+1}\right.} \delta_{i_{1}}^{b_{1}} \ldots \delta_{\left.i_{k}\right]}^{\left.b_{k}\right]}
$$

since the tensor is trace-free, we get

$$
0=T_{\left[a_{1} \ldots a_{k}\right.}^{i_{1} \ldots i_{k}} \delta_{\left.a_{k+1}\right]}^{\left[b_{k+1}\right.} \delta_{i_{1}}^{b_{1}} \ldots \delta_{i_{k}}^{\left.b_{k}\right]} .
$$

Absorbing the deltas gives the theorem.

(This proof shows that this second theorem is actually true for the weaker condition, $2 k \geq n$; but the validity of the second theorem when $2 k>n$ also follows from the first theorem, which has the more fundamental condition.)

The theorems immediately yield the familiar results in Example [I.2:

Example III.3. Theorem III.1 with $k=2$ applied to the trace-free $(2,2)$-form $W_{a b c d}$ gives $W_{a b c d}=0$ when $n \leq 3$. From Theorem $\left[I I .2\right.$ we find directly $W^{[c d}{ }_{[e f} \delta_{b]}^{a]}=0$ when $n \leq 4$.

An additional well-known result is found by multiplying this with $W^{e f}{ }_{c d}$ to get

$$
W_{d e}^{a c} W_{b c}^{d e}=\frac{1}{4} \delta_{b}^{a} W^{c d}{ }_{e f} W^{e f}{ }_{c d} .
$$

Multiplying with $W^{e f}{ }_{g a} W^{b g}$ cd instead yields the scalar identity cubic in $W^{a b}{ }_{c d}$,

$$
W^{a b}{ }_{c e} W^{c d}{ }_{a f} W^{e f}{ }_{b d}=\frac{1}{4} W^{a b}{ }_{c d} W^{c d}{ }_{e f} W^{e f}{ }_{a b} .
$$

The third result in Example II.2 may also be obtained - but in a more indirect manner; we consider the tensor $T^{a b e f}{ }_{c d k l}=W_{[a d}^{[a b} \delta_{k}^{e} \delta_{l]}^{f]}$ and by a direct calculation we can confirm its trace to be zero in five dimensions. Hence, from Theorem III.1, it follows immediately that $T^{a b e f} c d k l$ is identically zero in five dimensions.

The results in Example II.4 can also be obtained in the same way.

As well as showing how familiar identities were direct consequences of his theorems, Lovelock [19] also deduced some new interesting identities. More recently, additional important, and sometimes unexpected, identities have been shown to follow from Lovelock's theorems, [2, 7, 8, 10].

However, we emphasise again that the results in Example II.3 and II.4 cannot be deduced directly from Lovelock's theorems. 


\section{GENERAL THEOREMS ON DIMENSIONALLY DEPENDENT IDENTITIES}

The natural generalisation of Lovelock's theorems is the following theorem.

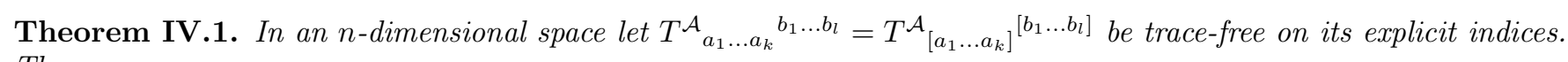
Then

$$
T_{\left[a_{1} \ldots a_{k}\right.}^{\mathcal{A}^{\left[b_{1} \ldots b_{l}\right.}} \delta_{a_{k+1}}^{b_{l+1}} \ldots \delta_{\left.a_{k+d}\right]}^{\left.b_{l+d}\right]}=0
$$

where $d \geq n-k-l+1$ and $d \geq 0$.

Proof. The proof is analogous to the proof of Theorem III.2. This time we antisymmetrise over $k+l+d \geq n+1$ indices to get the identity.

$$
0=T_{\left[a_{1} \ldots a_{k}\right.}^{i_{1} \ldots i_{l}} \delta_{a_{k+1}}^{b_{l+1}} \ldots \delta_{a_{k+d}}^{b_{l+d}} \delta_{i_{1}}^{b_{1}} \ldots \delta_{\left.i_{l}\right]}^{b_{l}}=T_{\left[a_{1} \ldots a_{k}\right.}^{\mathcal{A}_{1} \ldots i_{l}} \delta_{a_{k+1}}^{b_{l+1}} \ldots \delta_{a_{k+d}}^{b_{l+d}} \delta_{i_{1}}^{b_{1}} \ldots \delta_{\left.i_{l}\right]}^{\left.b_{l}\right]} .
$$

Since the tensor $T$ is trace-free, we get

$$
0=T^{\mathcal{A}}{ }_{\left[a_{1} \ldots a_{k}\right.}^{i_{1} \ldots i_{l}} \delta_{a_{k+1}}^{\left[b_{l+1}\right.} \ldots \delta_{\left.a_{k+d}\right]}^{b_{l+d}} \delta_{i_{1}}^{b_{1}} \ldots \delta_{i_{l}}^{\left.b_{l}\right]} .
$$

Absorbing the deltas gives the result.

Remarks.

- For completeness, we add, that if we adopt the convention that tensors with only lower or only upper indices are considered 'trace-free', then the above results also hold for the two classes of tensors where $k=0$ or $l=0$. In addition, for the case $k=0$ and $l=0$, i.e., $T$ a scalar, we simply get the trivial result of the vanishing of the Kronecker delta symbol with $n+1$ index pairs in $n$-dimensional space.

- The theorem generalises Lovelock's results by associating, in $n$-dimensional space, an identity with any tensor, since - no matter what its index configuration - part of it can be considered as a $(k, l)$-form, with the above conventions when $k=0$ or $l=0$. But it is for trace-free double forms with $k \neq 0 \neq l$ that it will be most useful. So when $l=0$ or $k=0$ and $d=0$ we have identity (8); when $k=l \neq 0$ and $d=0$ this is Theorem III.1 and when $k=l \neq 0$ and $d=1$ this is Theorem III.2. Had we wished only to generalise to the case $k=l$ for any $d$, we could have used Theorem III.1 directly in our proof, as in Example II.2.

- No stronger result on $T$ can be obtained by taking the trace of (33), since then the left hand side just collapses to zero. In addition, the discussion at the end of Section II would strongly suggest that the conditions on $d$ can not be relaxed for non-zero $T$; we shall confirm later in Theorem [V.5 that this is indeed so.

- Of course this theorem does not mean that we cannot construct results beginning with double forms which are not trace-free. Rather, what will happen is that if we begin with such a tensor we will get an apparently more complicated identity with explicit trace terms; if the double form is then decomposed into trace-free and trace parts, the resulting simplification will leave us with the identity which would be obtained by beginning with the trace-free part of the double form.

We shall now illustrate the relevance of the new results in Theorem IV.1 to familiar tensors as follows,

Example IV.2. Applying Theorem IV.1 to the trace-free $(2,1)$-form $L_{a b}{ }^{c}(l=1, k=2)$ in dimensions $n=3$ with $d=1$ gives the identity

$$
L_{[a b}^{[d} \delta_{c]}^{e]}=0
$$

and in dimensions $n=4$, with $d=2$

$$
L_{[a b}{ }^{[e} \delta_{c}^{f} \delta_{d]}^{g]}=0
$$

For trace-free $(2,2)$-forms $(l=k=2)$ in dimensions $n=5$ with $d=2$ gives the identity

$$
W_{[a b}^{[e f} \delta_{c}^{g} \delta_{d]}^{h]}=0 .
$$

For trace-free $(1,1)$-forms $S^{a}{ }_{b}(l=k=1)$, in dimensions $n=4$ with $d=3$ gives the identity

$$
S_{[a}^{[e} \delta_{b}^{f} \delta_{c}^{g} \delta_{d]}^{h]}=0
$$


Analogous basic identities can be found in other dimensions, and all such identities can then be exploited to build up other useful important identities, as we shall demonstrate in the next section.

We now consider whether we can generalise these results in another manner, by asking whether any kind of converses exist. Firstly, by way of example, we consider the identity (38) obtained from Theorem IV.1 for the trace-free $(2,2)$ forms when $n \leq 5$. As mentioned in the third remark above, we would like to confirm explicitly that the stronger condition $W_{[a b}^{[d e} \delta_{c]}^{f]}=0$, which holds in four dimensions, does not hold in dimensions $n=5$. A related task would be to confirm explicitly that the same identity (38) as holds in $n \leq 5$ dimensions does not hold in higher dimensions. We shall see from Theorem IV.5 that we can confirm explicitly an even stronger version of these results.

Secondly, with respect to the same example, a natural question to ask is whether the trace-free condition is also a necessary condition, i.e., whether any forms with non-zero trace can satisfy this identity $W_{[a b}^{[e f} \delta_{c}^{g} \delta_{d]}^{h]}=0$ in dimensions $n \leq 5$. Whether an alternative $(2,2)$-form - lacking the trace-free properties but perhaps with different symmetry properties - can satisfy the basic identity is not obviously ruled out. As an example, we could ask whether a $(2,2)$-form like the Riemann tensor $R_{a b}{ }^{c d}$ - lacking the trace-free properties of $W_{a b}{ }^{c d}$, but having the additional properties $R_{a[b c d]}=0$ and $R_{a b c d}=R_{c d a b}$ - can satisy the identities. However, we shall see from Theorem IV.6 that only trace-free $(2,2)$-forms satisfy the identity (38) in dimensions $n \leq 5$.

We first present a lemma which is then used in the proof of the two theorems. We emphasise that this lemma is also useful in its own right, and we shall demonstrate how it can be viewed as a generalisation of a familiar result for the Kronecker delta.

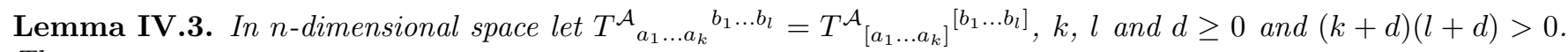
Then

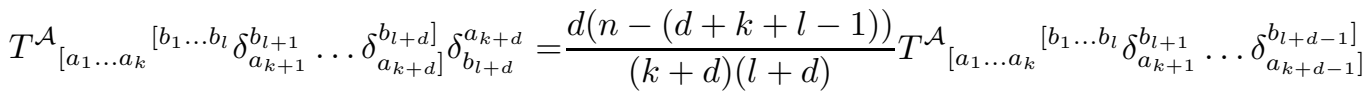

$$
\begin{aligned}
& +\frac{(-1)^{k+l} k l}{(k+d)(l+d)} T_{c\left[a_{1} \ldots a_{k-1}\right.}^{\mathcal{A}}{ }^{c\left[b_{1} \ldots b_{l-1}\right.} \delta_{a_{k}}^{b_{l}} \ldots \delta_{\left.a_{k+d-1}\right]}^{\left.b_{l+d-1}\right]} .
\end{aligned}
$$

Proof. If $k, l$ or $d$ is zero the lemma is trivial. Assume they are non-zero.

On the left hand side the last two deltas combine to give

$$
T^{\mathcal{A}}{ }_{\left[a_{1} \ldots a_{k}\right.}{ }^{\left[b_{1} \ldots b_{l}\right.} \delta_{a_{k+1}}^{b_{l+1}} \ldots \delta_{a_{k+d-1}}^{b_{l+d-1}} \delta_{c]}^{c]} .
$$

By summing over all possible positions of the dummy indices $c$ we get

$$
\frac{(k+d-1) !(l+d-1) !}{(k+d) !(l+d) !} \sum_{i, j} c_{i j} T_{\left[a_{1} \ldots a_{k}\right.}^{\left[b_{1} \ldots b_{l}\right.} \delta_{a_{k+1}}^{b_{l+1}} \ldots \delta_{|c|}^{b_{k_{i}}} \ldots \delta_{a_{l_{j}}}^{|c|} \ldots \delta_{\left.a_{k+d-1}\right]}^{\left.b_{l+d-1}\right]}
$$

where $c_{i j}= \pm 1$ depending on whether the index configuration is an even or an odd permutation.

It is now clear that we get only the two types of terms that are on the right hand side of the theorem; the last type when both dummy indices are on $T$ and the other type when at least one of them is on a $\delta$. What remains is to confirm the coefficients, which essentially means counting how many of each kind occurs and what sign they have.

When both dummy indices are on $T$ they can be moved to the first position. That means that they have moved a total of $(k+d-1)+(l+d-1)$ positions and changed sign equally many times. That gives a factor $(-1)^{k+l}$. There are a total of $k l$ such terms.

When both dummy indices are on the same $\delta$ they have moved equally many positions; thus such terms are added. There are $d$ such terms and we also get a factor $n$ since $\delta_{i}^{i}=n$.

When both dummy indices are on different $\delta$ one of them can be absorbed thereby distorting the order of the other indices. Once that order is restored we have overall an odd permutation; thus such terms are subtracted. There is a total of $d(d-1)$ such terms.

The same situation occurs when one of the dummy indices is on a $\delta$ and the other one is on $T$. There are $l d$ such terms with the upper index on $T$ and $k d$ with the lower index on $T$.

Taking all this together gives the identity in the lemma.

When we consider $k=0$ and $l=0$, with $T$ a non-zero constant, in Lemma IV.3 we get a familiar identity for the Kronecker delta,

Corollary IV.4. In n-dimensional space

$$
\delta_{\left[a_{1}\right.}^{\left[b_{1}\right.} \ldots \delta_{\left.a_{d}\right]}^{\left.b_{d}\right]} \delta_{b_{d}}^{a_{d}}=\frac{(n-d+1)}{d} \delta_{\left[a_{1}\right.}^{\left[b_{1}\right.} \ldots \delta_{\left.a_{d-1}\right]}^{\left.b_{d-1}\right]} .
$$


We now use Lemma IV.3 to establish the two theorems.

Theorem IV.5. In an n-dimensional space let $T^{\mathcal{A}}{ }_{a_{1} \ldots a_{k}} b_{1} \ldots b_{l}=T^{\mathcal{A}}{ }_{\left[a_{1} \ldots a_{k}\right]}\left[b_{1} \ldots b_{l}\right]$. If

$$
T^{\mathcal{A}}{ }_{\left[a_{1} \ldots a_{k}\right.}^{\left[b_{1} \ldots b_{l}\right.} \delta_{a_{k+1}}^{b_{l+1}} \ldots \delta_{\left.a_{k+d}\right]}^{\left.b_{l+d}\right]}=0
$$

then

$$
n>d+k+l-1 \Longrightarrow T^{\mathcal{A}}{ }_{a_{1} \ldots a_{k}}^{b_{1} \ldots b_{l}}=0 .
$$

Proof. The basic idea is to repeatedly take traces of equation (44) getting a sequence of equations; in the last of these no $\delta$ remains. The result follows from substituting equations at the end of this sequence into earlier ones. However, care must be taken so that no unwanted cancelling of terms occur which forces this process to stop prematurely.

To make the proof easier to overview we define the following notation. Let $(d, k, l)=T^{\mathcal{A}}{ }_{\left[a_{1} \ldots a_{k}\right.}\left[b_{1} \ldots b_{l} \delta_{a_{k+1}}^{b_{l+1}} \ldots \delta_{\left.a_{k+d}\right]}^{\left.b_{l+d}\right]}\right.$ and if $k$ and $l$ are less then the actual number of indices on $T$ then we contract over the remaining ones. Thus Lemma IV.3 can be written as the trace of $(d, k, l)=\frac{d(n-(d+k+l-1))}{(k+d)(l+d)}(d-1, k, l)+\frac{(-1)^{k+l} k l}{(k+d)(l+d)}(d, k-1, l-1)$ and $T$ itself as $(0, k, l)$ and the trace of $T$ as $(0, k-1, l-1)$.

We observe that the coefficients in Lemma IV.3 are non-zero if $d$ is non-zero and $k$ and $l$ are non-zero respectively.

First assume that $k+l$ is even, then the coefficients in Lemma IV.3 are non-negative. Start with equation (44) and multiply with $\delta_{b_{l+d}}^{a_{k+d}}$ and use Lemma IV.3. Then we get $0=(d-1, k, l)+(d, k-1, l-1)$ where we have omitted the coefficients.

Doing that once more gives us $0=(d-2, k, l)+(d-1, k-1, l-1)+(d, k-2, l-2)$. Repeating this process gives a sequence of equations as illustrated in Figure 1 where each row corresponds to one equation.

The last equation is $0=(0, k-l, 0)$ (assuming $k \geq l)$. Putting this into the second last equation gives $0=$ $(0, k-l+1,1)$ by using that $0=(j, k, l) \Longrightarrow 0=(j+1, k, l)$. Feeding the new information into earlier equations gives the desired conclusion $0=(0, k, l)$.

If $k+l$ is odd then there is a minus sign in Lemma IV.3 which means that there is a risk of cancellation in the above process. However, checking how the signs propagate gives us Figure 2 where the sign at a node is the sign of the term and the sign at the edge is the sign of the coefficient in the identity in Lemma IV.3.

It is now clear that there will be no cancellations since each term originates as the difference between two terms with different sign.

Theorem IV.6. In an n-dimensional space let $T^{\mathcal{A}}{ }_{a_{1} \ldots a_{k}}{ }^{b_{1} \ldots b_{l}}=T^{\mathcal{A}}{ }_{\left[a_{1} \ldots a_{k}\right]}\left[b_{1} \ldots b_{l}\right]$ where $n \geq k+l-1$ and let $d=$ $n-k-l+1$. Then

$$
T_{\left[a_{1} \ldots a_{k}\right.}^{{ }^{\left[b_{1} \ldots b_{l}\right.}} \delta_{a_{k+1}}^{b_{l+1}} \ldots \delta_{\left.a_{k+d}\right]}^{\left.b_{l+d}\right]}=0 \Longleftrightarrow T_{a_{1} \ldots a_{k}}^{\mathcal{A}}{ }^{b_{1} \ldots b_{l}} \text { is trace-free on its explicit indices. }
$$

Proof. $\Longleftarrow:$ Follows directly from Theorem IV.1

$\Longrightarrow$ : The case $d=0$ is trivial. Assume $d>0$. Contracting

$$
0=T_{\left[a_{1} \ldots a_{k}\right.}^{\left[b_{1} \ldots b_{l}\right.} \delta_{a_{k+1}}^{b_{l+1}} \ldots \delta_{\left.a_{k+d}\right]}^{\left.b_{l+d}\right]}
$$

once and using Lemma IV.3 gives

$$
0=T^{\mathcal{A}}{ }_{i\left[a_{1} \ldots a_{k-1}\right.}{ }^{i\left[b_{1} \ldots b_{l-1}\right.} \delta_{a_{k}}^{b_{l}} \ldots \delta_{\left.a_{k+d-1}\right]}^{\left.b_{l+d-1}\right]} .
$$

The theorem now follows from Theorem IV.5 applied to the tensor

$$
\tilde{T}_{a_{1} \ldots a_{k-1}}{ }^{b_{1} \ldots b_{l-1}}=T^{\mathcal{A}}{ }_{i a_{1} \ldots a_{k-1}} i b_{1} \ldots b_{l-1} .
$$

Example IV.7. We know that for the Weyl curvature tensor $C_{a b c d}$ (a trace-free symmetric $(2,2)$-form), $C_{[a b}\left[e f \delta_{c}^{g} \delta_{d]}^{h]}=\right.$ 0 in dimensions $n \leq 5$. From Theorem IV.5 we can conclude that this tensor (or indeed any non-zero $(2,2)$-form) cannot satisfy the stronger condition $C_{[a b}{ }^{[d e} \delta_{c]}^{f]}=0$ in dimensions $n \geq 5$; in addition we can conclude that there are absolutely no non-zero $(2,2)$-forms $T_{a b}{ }^{c d}$ satisfying $T_{[a b}{ }^{[e f} \delta_{c}^{g} \delta_{d]}^{h]}=0$ in dimensions greater than five.

From Theorem IV.6 we can conclude that there are no $(2,2)$-forms $R_{a b}{ }^{c d}$ with non-zero trace (e.g., a Riemann tensor) satisfying the identity $R_{[a b}{ }^{[e f} \delta_{c}^{g} \delta_{d]}^{h]}=0$ in dimensions $n=5$. 
The trace in Lemma IV.3 is not the only trace that is possible for expressions of the type we are investigating. For completeness we here present the other possibility.

Lemma IV.8. Let $T^{\mathcal{A}}{ }_{a_{1} \ldots a_{k}} c b_{1} \ldots b_{l}=T^{\mathcal{A}}{ }_{\left[a_{1} \ldots a_{k}\right]}^{c\left[b_{1} \ldots b_{l}\right]}, k$ and $d \geq 0$ and $k+d>0$. Then

$$
\begin{aligned}
T_{\left[a_{1} \ldots a_{k}\right.}^{{ }^{\mathcal{A}}\left[b_{1} \ldots b_{l}\right.} \delta_{a_{k+1}}^{b_{l+1}} \ldots \delta_{\left.a_{k+d}\right]}^{\left.b_{l+d}\right]} \delta_{c}^{a_{k+d}}= & \frac{d(-1)^{l+d-1}}{k+d} T^{\mathcal{A}}{ }_{\left[a_{1} \ldots a_{k}\right.}\left[b_{1} \ldots b_{l} b_{l+1} \delta_{a_{k+1}}^{b_{l+2}} \ldots \delta_{a_{k+d}+1}^{\left.b_{l+d}\right]}\right. \\
& +\frac{k(-1)^{k+d-1}}{k+d} T^{\mathcal{A}}{ }_{c\left[a_{1} \ldots a_{k-1}\right.}{ }_{c\left[b_{1} \ldots b_{l}\right.} \delta_{a_{k}}^{b_{l+1}} \ldots \delta_{\left.a_{k+d-1}\right]}^{\left.b_{l+d}\right]}
\end{aligned}
$$

Proof. If $k$ or $d$ is zero the lemma is trivial. Assume they are non-zero.

Absorbing the last delta on the left hand side gives

$$
T^{\mathcal{A}}{ }_{\left[a_{1} \ldots a_{k}\right.}{ }^{c\left[b_{1} \ldots b_{l}\right.} \delta_{a_{k+1}}^{b_{l+1}} \ldots \delta_{c]}^{\left.b_{l+d}\right]}
$$

By summing over all possible positions of the lower dummy index $c$ we get

$$
\frac{(k+d-1) !}{(k+d) !} \sum_{i} c_{i} T^{\mathcal{A}}{ }_{\left[a_{1} \ldots a_{k}\right.}{ }^{c\left[b_{1} \ldots b_{l}\right.} \delta_{a_{k+1}}^{b_{l+1}} \ldots \delta_{|c|}^{b_{i}} \ldots \delta_{\left.a_{k+d-1}\right]}^{\left.b_{l+d}\right]}
$$

where $c_{i}= \pm 1$ depending on whether the index configuration is an even or an odd permutaion.

When the $c$ is on a delta it can be absorbed giving terms of the same type as the first term on the right hand side of (50). There are $d$ such terms and on each of them the indices have been moved a total of $l+d-1$ steps.

When the $c$ is on $T$ it can be moved to the first position of the lower indices giving terms of the same type as the second term on the right hand side of (50). There are $k$ such terms and on each of them the index has been moved a total of $k+d-1$ steps.

\section{APPLiCATiONS.}

In the last sections we have noted that, associated with each tensor, are a number of fundamental identities; and it is from such identities that more involved and more subtle identities can be constructed, which in turn yield familiar identities. So, we shall now exploit our results in two particular types of applications:

\section{A. Identities involving scalar invariants of Riemann tensors.}

Relationships between scalar invariants of tensors (such as (2), (3) and (32)) play a very important role in classical invariant theory, as well as in many practical applications. For instance, the study of the scalar invariants of the Riemann tensor in four dimensions has posed many interesting problems, which have not all been resolved 44, 11, 14, 16, 20, 26, 27, 30. The theorems given in this paper provide important tools for a systematic study of invariants of the Weyl, trace-free Ricci and Lanczos tensors. However, now we shall just apply our results to some representative examples.

Example V.1. Although we can form different scalars from two $(2,2)$-forms $W_{a b} c d$, in four dimensions, the simplest basic identity is $W^{[c d}{ }_{[e f} \delta_{b]}^{a]}=0$ (with 6 free indices), and so we can immediately see that it cannot yield relationships between scalar invariants involving only two $(2,2)$-forms.

However, we already know that multiplying this identity by $W^{e f}{ }_{g a} W^{b g}{ }_{c d}$ gives us the scalar identity (32), which yields a relationship between some cubic scalar invariants; while multiplying by other quadratic terms will give different relationships between different cubic scalar invariants. Clearly we can also choose various suitable expressions involving three $(2,2)$-forms, which will yield scalar identities of fourth order when multiplied with $W^{[c d}\left[f^{[} \delta_{b]}^{a]}=0\right.$. Hence, it is possible to investigate, for each order, all such possible relationships between all scalar invariants of that order.

When we consider higher dimensions we have analogous basic identities. In five dimensions the simplest basic identity is $W_{[a b}{ }^{[e f} \delta_{c}^{g} \delta_{d]}^{h]}=0$ (with eight free indices), and hence the lowest order where we can get relationships between scalar invariants from this identity is also at third order; while in six dimensions with the simplest basic identity having 10 free indices there can exist no relationships between scalar invariants at third order coming from this identity. These results can be applied to the Weyl tensor, where they may simplify a little because of its extra symmetries. 
It is interesting to note that Dianyan Xu's work [6] was motivated by a concern that non-trivial relationships might exist between counterterms in the Lagrangian (essentially invariant scalars constructed from products of the Riemann tensor) of a renormalizable quantum field theory; in particular, if his identities (2), (3), or any other cubic scalar identities, are true in six dimensions then work by Jack and Parker 15] would need to be revaluated. However, Jack and Parker [16] have subsequently shown explicitly that no such third order identities can exist in six dimensions; our result in the example above agrees with this. Jack and Parker 16 have conjectured that in $2 n$ dimensions there do not exist any identities between Riemann scalars of order $n$; we shall show how our results relate to this conjecture in a subsequent paper.

Example V.2. In order to confirm that the second identity found in [6] was a four dimensional one, Harvey first established the intermediate third order identity (5) by antisymmetrising over six indices and this intermediate identity is therefore valid in five as well as in four dimensions; in our previous example we noted that five dimensions was the lowest dimension where such third order identities could be constructed for the Weyl tensor. However, one would suspect that the second four dimensional identity (3) could be obtained directly; this is confirmed by using the four dimensional identity $C^{[c d}{ }_{[e f} \delta_{b]}^{a]}=0$ and expanding $C^{e f}{ }_{a i} C^{i b}{ }_{c d} C^{[c d}{ }_{[e f} \delta_{b]}^{a]}=0$. It can also be obtained, in the manner of Harvey, by antisymmetrising over 5 indices and expanding

$$
R_{[a b}^{a b} R_{c d}^{e f} R_{e] f}^{c d}=0 .
$$

Example V.3. An important application of the identity in Example IV.2 is to find relationships between scalar invariants of the Lanczos potential [17] in four dimensions. Since the identity $L_{[a b}\left[{ }^{e} \delta_{c}^{f} \delta_{d]}^{g]}=0\right.$ has seven free indices, the lowest order relationship between scalar invariants that we can obtain from it is of order four. For example, by expanding

$$
0=L_{[a b}{ }^{[e} \delta_{c}^{f} \delta_{d]}^{g]} L_{e}^{a b} L_{f g}{ }^{h} L^{c d}{ }_{h}
$$

we obtain the relationship

$$
0=L_{a b c} L^{a b c} L_{d e f} L^{d e f}+L_{a b c} L^{a b f} L_{d e f} L^{d e c}-4 L_{a f c} L^{a e c} L_{d e h} L^{d f h}-2 L_{a b c} L^{a b d} L_{d e f} L^{c e f}-4 L_{a b c} L^{a d} L^{b f h} L_{d h}^{c} .
$$

This of course will not be the only relationship; we could instead multiply the original identity by $L^{a b}{ }_{e} L_{f h}{ }^{c} L_{g}{ }^{h d}$. Therefore, we can investigate all possible contractions of the original identity with three Lanczos tensors and find the corresponding relationship between all possible quartic Lanczos scalars in a systematic manner.

There is an important caveat in the above examples. By a systematic study we are able to obtain all relationships between scalar invariants of a particular order which arise from our dimensionally dependent identities. Of course, we would like to be able to conclude that we have obtained all possible such relationships. Recent work on invariant theory by Gover 13 links relationships involving scalar invariants of tensors in $n$ dimensions to antisymmetrising over $n+1$ indices. Although this does not, at this stage, enable us to conclude that all relationships between scalar invariants originate from our dimensionally dependent identities, it would lead us to believe that the results in this paper will be useful in such difficult tasks as determining complete and independent sets of scalar invariants, and their syzygies.

\section{B. Simplifying complicated expressions in four dimensions.}

There have recently been situations [2, 7, 8, 10] where rather complicated tensor expressions in $n$ dimensions have been shown, unexpectedly, to be identically zero when specialised to four dimensions, via Lovelock's theorems. So we would anticipate that the more general results obtained in this paper should also be useful in simplifying such expressions, and we now give some examples.

Example V.4. In [2] Lovelock's identities were used to show that, when the wave equation for the Weyl tensor is constructed from the Bianchi identities, the sum of terms which involve products of Weyl and trace-free Ricci tensors disappeared in four, and only four, dimensions, because from the 4-dimensional identity $C_{[a b}{ }^{[d e} \delta_{c]}^{f]}=0$ we can obtain $C_{[a b}^{[d e} \delta_{c]}^{f]} \tilde{R}_{f}^{c}=0$ whose left hand side, when expanded, is precisely this sum of terms. 
We shall now show that the analogous component of the Bel tensor [3] disappears in four dimensions by virtue of the same identity. The Bel tensor is given in $n$ dimensions by

$$
B_{a b c d}=R_{a e c f} R_{b}{ }^{e}{ }^{f}+R_{a e d f} R_{b}{ }^{e}{ }^{f}-\frac{1}{2} g_{a b} R_{e f c g} R^{e f} d^{g}-\frac{1}{2} g_{c d} R_{a e f g} R_{b}{ }^{e f g}+\frac{1}{8} g_{a b} g_{c d} R_{e f g h} R^{e f g h} .
$$

When the standard decomposition is substituted we obtain [5]

$$
B_{a b c d}=\mathcal{T}_{a b c d}+\mathcal{Q}_{a b c d}+\mathcal{M}_{a b c d}
$$

where $\mathcal{T}_{a b c d}$ is the Bel-Robinson tensor consisting of quadratic terms in the Weyl tensor, $\mathcal{M}_{a b c d}$ consists of quadratic terms in the Ricci tensor $R_{a b}$, and $\mathcal{Q}_{a b c d}$ consists of products of Weyl and Ricci components (gravity-matter coupling term) 25],

$$
\begin{aligned}
\mathcal{Q}_{a b c d}= & \frac{1}{n-2}\left(-4 C^{i}{ }_{(c d)(a} \tilde{R}_{b) i}-4 C^{i}{ }_{(a b)(c} \tilde{R}_{d) i}+2 \tilde{R}_{i j}\left(C_{a}{ }^{i}\left({ }^{j} g_{d) b}-C_{c}{ }^{i} d^{j} g_{a b}+C_{b}{ }^{i}\left(c^{j} g_{d) a}-C_{a}{ }^{i}{ }^{j} g_{c d}\right)\right)\right.\right. \\
& +\frac{2}{n(n-1)} R\left(C_{a c b d}+C_{a d b c}\right) .
\end{aligned}
$$

The structure of $\mathcal{Q}$ (maximum of one delta) suggests that we investigate the four dimensional identity $C_{[a c}{ }^{[b d} \delta_{e]}^{f]}=0$; when we multiply this identity by $\tilde{R}_{e}^{f}$ we obtain the identity

$$
C_{d i j[a} g_{c] b} \tilde{R}^{i j}-C_{b i j[a} g_{c] d} \tilde{R}^{i j}+C_{a c i[b} \tilde{R}_{b]}^{i}+C_{b d i[a} \tilde{R}_{c]}^{i}=0 .
$$

By symmetrising over the index pair $(c d)$ we get precisely the identity

$$
4 C^{i}{ }_{(c d)(a} \tilde{R}_{b) i}+4 C^{i}{ }_{(a b)(c} \tilde{R}_{d) i}-2 \tilde{R}_{i j}\left(C_{a}{ }^{i}\left({ }^{j}{ }^{j} g_{d) b}-C_{c}{ }^{i} d^{j} g_{a b}+C_{b}{ }^{i}{ }_{\left(c^{j}\right.} g_{d) a}-C_{a b^{j}}{ }^{i} g_{c d}\right)=0\right.
$$

and so the gravity-matter coupling term (58) simplifies, in four dimensions, to

$$
\mathcal{Q}_{a b c d}=R\left(C_{a c b d}+C_{a d b c}\right) / 6 .
$$

Bonilla and Senovilla [5] have obtained this result, but since they were working with duals, they did not encounter the four dimensional identity explicitly; Zund [31] has also found the remarkably simple gravity-matter coupling term, using spinors.

The possibility of the five dimensional identity $C_{[a b}\left[c d \delta_{e}^{f} \delta_{h]}^{g]}=0\right.$ supplying significant simplification is obviously ruled out since, after multiplication with one trace-free Ricci tensor, there will still be at least one term with two deltas.

Example V.5. The four dimensional identity for the Lanczos potential $L_{a b}{ }^{c}$,

$$
2 L^{\text {def }} g_{[a|c|} C_{b] d e f}-2 L_{[a}{ }^{d e} C_{b] e d c}-\frac{1}{2} L^{d e}{ }_{c} C_{d e a b}=0
$$

plays an important role in the derivation of the wave equation of the Lanczos potential [9]. Its existence was first noted because the spinor equivalence of the left hand side collapsed; subsequently, it was proven by Edgar in [7] by using four dimensional duals, and also by using Lovelock's four dimensional identity $C_{[a b}{ }^{[c d} \delta_{e]}^{f]}=0$. We can also deduce it by using the four dimensional identity in Example IV.2, and then expanding

$$
L_{[a b}{ }^{[c} \delta_{d}^{f} \delta_{e]}^{g]} C_{f g}^{d e}=0 .
$$

The remaining examples involve identitities which cannot be deduced directly from Lovelock's identities.

Example V.6. In [4], Bonanos demonstrated that a complicated tensor

$$
\begin{aligned}
\chi_{a b c d}^{\prime}= & \tilde{R}_{a c} \tilde{R}_{b}{ }^{m} \tilde{R}_{m d}+\tilde{R}_{b d} \tilde{R}_{a}{ }^{m} \tilde{R}_{m c}-\tilde{R}_{a d} \tilde{R}_{b}{ }^{m} \tilde{R}_{m c}-\tilde{R}_{b c} \tilde{R}_{a}^{m} \tilde{R}_{m d}+\tilde{R}_{a}^{m} \tilde{R}_{m}^{n} \tilde{R}_{n c} g_{b d}+\tilde{R}_{b}{ }^{m} \tilde{R}_{m}{ }^{n} \tilde{R}_{n d} g_{a c} \\
& -\tilde{R}_{a}{ }^{m} \tilde{R}_{m}{ }^{n} \tilde{R}_{n d} g_{b c}-\tilde{R}_{b}{ }^{m} \tilde{R}_{m}{ }^{n} \tilde{R}_{n c} g_{a d} \\
& -\frac{1}{2}\left(\tilde{R}_{m n} \tilde{R}^{m n}\right)\left(\tilde{R}_{a c} g_{b d}+\tilde{R}_{b d} g_{a c}-\tilde{R}_{a d} g_{b c}-\tilde{R}_{b c} g_{a d}\right)-\frac{1}{3}\left(\tilde{R}_{m n} \tilde{R}^{m r} \tilde{R}_{r}^{n}\right)\left(g_{a c} g_{b d}-g_{a d} g_{b c}\right)
\end{aligned}
$$


of third order in the trace-free Ricci tensor $\tilde{R}_{a b}$, which had been used in the study of Riemann invariants in four dimensions [30], was in fact, surprisingly, identically zero.

In [8] it was demonstrated how this result could be seen as an indirect consequence of Lovelock's results [19]. By applying Theorem III.2 to the trace-free Plebanski tensor $P_{a b}{ }^{c d}$ which is the 'square' of the Ricci tensor given by

$$
P_{a b}{ }^{c d}=2 \tilde{R}_{[a}^{[c} \tilde{R}_{b]}{ }^{d]}+2 \tilde{R}_{[a}{ }^{i} \tilde{R}_{|i|}{ }^{[c} \delta_{b]}^{d]}-\frac{1}{3} \tilde{R}_{j}{ }^{i} \tilde{R}_{i}{ }^{j} \delta_{[a}^{c} \delta_{b]}^{d}
$$

the following identity of second order in the trace-free Ricci tensor was obtained

$$
2 \tilde{R}_{[a}^{\left[{ }^{c}\right.} \tilde{R}_{b}{ }^{d} \delta_{e]}^{f]}+2 \tilde{R}_{[a}{ }^{i} \tilde{R}_{|i|}{ }^{[c} \delta_{b}^{d} \delta_{e]}^{f]}-\frac{1}{3} \tilde{R}_{j}{ }^{i} \tilde{R}_{i}^{j} \delta_{[a}^{c} \delta_{b}^{d} \delta_{e]}^{f}=0
$$

It was then shown in [8] that the identically zero tensor $\chi_{a b c d}^{\prime}$ found by Bonanos was just a direct consequence of multiplying the identity (66) by $\tilde{R}_{f}{ }^{e}$.

But we now have the complete picture. The basic identity in four dimensions for the trace-free Ricci tensor is the first order identity (39)

$$
\tilde{R}_{[a}^{[c} \delta_{b}^{d} \delta_{e}^{f} \delta_{g]}^{h]}=0
$$

By successive multiplications by the trace-free Ricci tensor we obtain, first of all, the identity (66) which is second order in the trace-free Ricci tensor; subsequently by multiplying the left hand side of $(67)$ by $\tilde{R}_{f}^{e} R_{h}{ }^{g}$ we obtain the third order identity which is $\chi_{a b c d}^{\prime}$ identically zero, and finally the fourth order identity, which is the Cayley-Hamilton theorem for the matrix representation of $\tilde{R}_{a}{ }^{b}$ in four dimensions, as shown in [8].

Example V.7. The Bel-Robinson tensor [24] is given in $n$ dimensions by

$$
\mathcal{T}_{a b c d}=C_{a e c f} C_{b}{ }_{d}{ }^{f}+C_{a e d f} C_{b}{ }^{e}{ }^{f}-\frac{1}{2} g_{a b} C_{e f c g} C^{e f}{ }_{d}^{g}-\frac{1}{2} g_{c d} C_{a e f g} C_{b}{ }^{e f g}+\frac{1}{8} g_{a b} g_{c d} C_{e f g h} C^{e f g h} .
$$

It is obviously symmetric over the first and last pair of indices, but in order to investigate its symmetry over all indices we need to examine,

$$
\mathcal{T}_{a[b c] d}=\frac{1}{4} C_{a d e f} C_{b c}{ }^{e f}-C_{e a f[b} C_{c]}{ }^{e}{ }^{f}-C_{f g e[a} g_{d][b} C_{c]}{ }^{e f g}+\frac{1}{8} g_{a[b} g_{c] d} C_{e f g h} C^{e f g h} .
$$

Its structure (maximum of two deltas) suggests that we investigate the five dimensional identity $C_{[b c}^{[a d} \delta_{e}^{g} \delta_{f]}^{h]}=0 ;$ by multiplying with $C_{g h}$ ef we obtain precisely the right hand side of (69). So the Bel-Robinson tensor is completely symmetric in both four and five dimensions, but of course this calculation does not give us any information about higher dimension. (Although we know that $C_{[a b}\left[c d \delta_{g}^{e} \delta_{h]}^{f]}=0\right.$ is not an identity in higher dimensions, we are considering the more complicated expression (69).) However, by taking the non trivial double trace on (69) we obtain

$$
\mathcal{T}^{a}{ }_{[a b]}^{b}=\frac{(n-4)(n-5)}{16} C_{a b c d} C^{a b c d}
$$

which shows that the dimensions four and five are both necessary and sufficient conditions for the Bel-Robinson tensor to be completely symmetric.

The fact that $\mathcal{T}_{a b c d}$ is completely symmetric, in, and only in, dimensions four and five was confirmed by Senovilla 25 from its definition in terms of duals, but in a less direct manner, where each dimension was considered seperately.

Example V.8. The Bel-Robinson tensor (constructed from the Weyl tensor), discussed in the last example, has the wrong dimension for energy, so Roberts 23] has proposed instead using an analogous construction with the Lanczos potential of the Weyl tensor which has the correct dimensions for energy. He has suggested looking at the most general expression quadratic in the Lanczos potential, but if instead we use Senovilla's definition of super-energy tensor [25] we find the super-energy tensor associated with the Lanczos potential $L_{a b}{ }^{c}$ (a trace-free $(2,1)$-form) is given in $n$ dimensions by

$$
\mathcal{T}_{a b c d}^{L}=L_{a e c} L_{b}{ }^{e}{ }_{d}+L_{a e d} L_{b}{ }^{e}{ }_{c}-\frac{1}{2} g_{a b} L_{e f c} L^{e f}{ }_{d}-g_{c d} L_{a e f} L_{b}{ }^{e f}+\frac{1}{4} g_{a b} g_{c d} L_{e f g} L^{e f g}
$$


It is obviously symmetric over the first and last pair of indices respectively. It is not symmetric over all indices which can be shown by choosing a local orthonormalised basis and $L_{131}=-L_{311}=-L_{232}=L_{322}=1$, all others zero in $n$ dimensions. Then $\mathcal{T}_{1[12] 2}^{L}= \pm \frac{1}{2}$ (the sign depends on the signature).

The Bel-Robinson tensor in the previous example had the pairwise symmetry $\mathcal{T}_{a b c d}=\mathcal{T}_{c d a b}$. That symmetry can be imposed on the super-energy tensor for the Lanczos potential by examining

$$
T^{L}{ }_{a b c d}=\frac{1}{2} \mathcal{T}_{a b c d}^{L}+\frac{1}{2} \mathcal{T}_{c d a b}^{L}
$$

instead. In order to investigate whether this makes it symmetric over all indices we examine

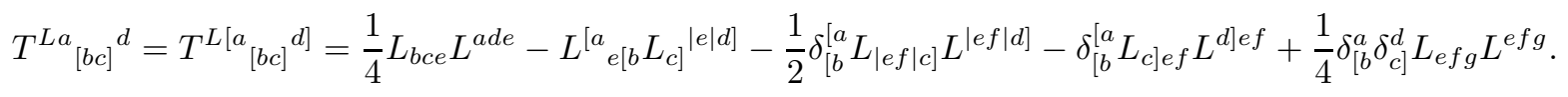

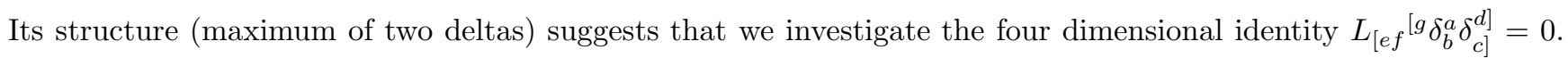
By multiplying with $L^{e f}$ we obtain the right hand side of $(73)$, so $T^{L}{ }_{a b c d}$ is completely symmetric in four dimensions and lower. It is, however, necessary to use the additional symmetry of the Lanczos potential $L_{[a b c]}=0$ so this result does not hold for any $(2,1)$-form.

By taking the non trivial double trace of 73 we obtain (by using $L_{[a b c]}=0$ again)

$$
T_{[a b]}^{L a}=\frac{(n-4)(n-3)}{8} L_{a b c} L^{a b c}
$$

which shows that the dimension being four or less is both a necessary and sufficient condition for the symmetrised super-energy tensor $T^{L}$ abcd for the Lanczos potential to be completely symmetric.

Although we can follow Senovilla's construction for the super energy tensor from any $(2,1)$-form $L_{a b}{ }^{c}$ in $n$ dimensions, we should point out that the Lanczos potential for the Weyl tensor is unlikely to exist generally in dimensions above four $[10$.

\section{SUMMARY AND DISCUSSION}

Of course, Lovelock's identities and our generalisations found in this paper are not really 'new' since they are just simple and direct specialisations of those fundamental identities found by antisymmetrising over $n+1$ indices in $n$ dimensions; furthermore, they are not complete alternatives for those fundamental identities, since, being their traces, they contain less information. Rather, the significance of these identities is that they highlight the fact that there exist heavily disguised versions of these fundamental identities when trace-free and antisymmetry properties are also introduced; and since we often are dealing with tensors with these explicit properties it is often the specialisations of the fundamental identities which are relevant in practical applications.

The use of dimensionally dependent identities is a powerful method which has been largely overlooked, perhaps because of its simplicity. A very striking example of this is to be seen in the algebraic Rainich condition for the electromagnetic energy tensor in four dimensions. Over the past 75 years this condition has been obtained by a variety of very different methods: Rainich 22 used invariant planes, while others have used duality rotations 28], complex duals [12, 29] and complicated matrix manipulation based on the Cayley-Hamilton theorem [1]; in spinors, a simple direct calculation has been given [21]. In fact Rainich's result was one of the motivations for Lovelock's work [19] and although he rederived the result using an explicit four dimensional identity, [18, 19, it is still a somewhat roundabout and contrived calculation. We shall now show that the result can be viewed as simply the four dimensional CayleyHamilton theorem when considered as a dimensionally dependent identity, or equivalently a trivial application of our basic result.

Example VI.1. The four dimensional algebraic Rainich identity associated with the energy momentum tensor $T^{a}{ }_{b}=$ $F_{c}^{a} F_{b}^{c}-\frac{1}{4} \delta_{b}^{a} F_{d}^{c} F_{c}^{d}$ of an electromagnetic field $F_{a b}$ is given by

$$
T_{c}^{a} T_{b}^{c}=\frac{1}{4} \delta_{b}^{a} T_{d}^{c} T_{c}^{d}
$$

When written out in full this identity is

$$
F_{c}^{a}{ }_{c} F_{d}^{c} F^{d}{ }_{e} F_{b}^{e}-\frac{1}{2} F^{a}{ }_{c} F^{c}{ }_{b} F^{d}{ }_{e} F^{e}{ }_{d}-\frac{1}{4} \delta_{b}^{a} F^{c}{ }_{d} F^{d}{ }_{e} F^{e}{ }_{f} F^{f}{ }_{c}+\frac{1}{8} \delta_{b}^{a} F^{c}{ }_{d} F^{d}{ }_{c} F^{e}{ }_{f} F^{f}{ }_{e}=0 .
$$


But this is simply the Cayley-Hamilton theorem for $F_{b}^{a}$ as given in Example I.2,

$$
F_{[c}^{c} F_{d}^{d} F_{e}^{e} F_{f}^{f} \delta_{b]}^{a}=0
$$

when specialised to antisymmetric $F_{a b}$. Equivalently the structure immmediately suggests specialising the four dimensional identity for trace-free $(1,1)$-forms $F^{\left[{ }_{[}{ }_{[c} \delta_{d}^{g} \delta_{e}^{h} \delta_{b]}^{a]}\right.}=0$ given in (39) to antisymmetric forms, from which we obtain the identity

$$
F^{[f}{ }_{[c} \delta_{d}^{g} \delta_{e}^{h} \delta_{b]}^{a]} F_{f}^{c} F_{g}^{d} F_{h}^{e}=0
$$

which, when expanded and specialised to antisymmetric $F_{a b}$, is identical to (76).

A more direct way to obtain $(76)$ is to expand

$$
F^{c d} F^{e f} F_{[c d} F_{e f} \delta_{b]}^{a}=0
$$

In this particular example, as in the others in the last section, we have been able to focus directly on the fundamental dimensionally dependent identity underlying the result. By identifying this underlying identity in our various applications to four dimensions in this paper, we are in a position to explore directly the possibility of generalisations to other dimensions and to other forms. We shall present such generalisations in a subsequent paper.

Of course, it is possible to establish results peculiar to four dimensions without having to deal explicitly with dimensionally dependent identities; this is most easily done by using spinors, where the dimension is built into the formalism. Indeed, it was the apparent discrepency between results in spinors and tensors which originally gave the clue to the existence of a number of these four dimensional identities. If we were only dealing with four dimensional spaces with Lorentz signature, then spinors would be the more efficient formalism to use; on the other hand, the advantage of the tensor formalism, is that once we have identified the underlying four dimensional tensor identity, generalisations to other dimensions can be sought.

In a similar way, when explicit four dimensional duals are used, then the dimension can also be built in by some of the identities satisfied by duals. For instance, the key identities associated with duals in work on super-energy tensors by Senovilla (equations (2) and (3) in [25]) have dimension $n$ built in explicitly; so these are dimensionally dependent identities constructed from identities of the form

$$
T_{\left[a_{1}\right.}^{\mathcal{A}} \eta_{\left.a_{2} a_{3} \ldots a_{n+1}\right]}=0 .
$$

In this context, it is significant that the four dimensional identity (31) is obtained in 25 essentially by taking double duals.

As emphasised in the last section, important applications of these results will be to Riemann tensors. Of course the Riemann tensor has additional symmetries to those of a $(2,2)$-form; these additional symmetries have not been explicitly considered in this paper, but would need to be considered explicitly in an exhaustive treatment of invariants of the Riemann tensor.

In conclusion, we emphasise that when examining what actually was required in the proofs of the theorems in Section 4 one discovers that very little structure was needed. Firstly, the results hold pointwise so the manifold structure is not needed. Secondly, there was no raising or lowering of indices so no assumption on what to use for that is needed; indeed not even the existence of such operations is needed. Thirdly, no metric was used. This means that those theorems can be applied to objects other than tensors: e.g. spinors when $n=2$; matrices when the number of indices on the objects are appropriate; or other indexed objects such as Christoffel symbols or tensors in coordinate index notation or tetrad index notation.

\section{ACKNOWLEDGMENTS}

SBE is grateful for support from the Swedish Natural Science Research Council.

\section{REFERENCES}

[1] Adler, R. et al. (1965). Introduction to general relativity, McGraw-Hill, London. 
[2] Andersson, F. and Edgar, S.B. (1996). The wave equations for the Weyl tensor/spinor and dimensionally dependent tensor identities, Int. J. of Mod. Phys D, 5, 217-225.

[3] Bel, L. (1962). Les états de radiation et le problème de l'énergie en relativité générale, Cahiers de Physique, 16, 59-80.

Reprinted in 2000. Radiation states and the problem of energy in general relativity, Gen. Rel. Grav., 32, 20472078.

[4] Bonanos, S. (1998). A new spinor identity and the vanishing of certain Riemann tensor invariants, Gen. Rel. Grav, 30, 653-658.

[5] Bonilla, M. Á. G. and Senovilla J. M. M. (1997). Some properties of the Bel and Bel-Robinson tensors, Gen. Rel. Grav, 29, 91-116 .

[6] Dianyan Xu. (1987). Two important invariant identities, Phys. Rev. D, 35, 769-770.

[7] Edgar, S. B. (1994). The wave equations for the Lanczos tensor/spinor, and a new tensor identity, Mod. Phys. Lett. A, 9, 479-482.

[8] Edgar, S. B. (1999). Four-dimensional tensor identities of low order for the Weyl and Ricci tensors, Gen. Rel. Grav., 31, 405-411.

[9] Edgar, S. B. and Höglund, A. (1997). The Lanczos potential for the Weyl curvature tensor: existence, wave equation and algorithms, Proc. R. Soc. Lond. A, 453, 835-851.

[10] Edgar, S. B. and Höglund, A. (2000). The Lanczos potential for the Weyl-Candidate tensors exists only in four dimensions, Gen. Rel. Grav., 32, 2307-2318.

[11] Fulling, S. A. et al. (1992). Normal forms for tensor polynomials: I. The Riemann tensor, C. Q. G., 9, 1151-1197.

[12] Goodinson, P. A. and Newing, R. A. (1968). Einstein-Maxwell fields with non-zero charge-current distribution, J. Inst. Maths Applics., 4, 270-275.

[13] Gover, A. R. (1997). The algebra of orthogonal group invariants, Queensland University of Technology, preprint.

[14] Harvey, A. (1995). Identities of the scalars of the four-dimensional Riemann manifold, J. Math. Phys. 36, 356-361.

[15] Jack, I. and Parker, L. (1985). Proof of summed form of proper-time expansion for propagator in curved spacetime, Phys. Rev. D., 31, 2439-2451.

[16] Jack, I. and Parker, L. (1987). Linear independence of renormalization counterterms in curved space-times of arbitrary dimensionality, J. Math. Phys., 28, 1137-1139.

[17] Lanczos, C. (1962). The splitting of the Riemann tensor, Rev. Mod. Phys., 34, 379-389.

[18] Lovelock, D. (1967). The Lanczos identity and its generalisations, Atti. Accad. Naz. Lincei (VIII)., 42, 187-194.

[19] Lovelock, D. (1970). Dimensionally dependent identities, Proc. Cambridge Philos. Soc., 68, 345-350.

[20] Ouchterlony, E. (1997). A systematic approach to matrix invariants, M.Sc. dissertation, LITH-MAT-Ex-97-14, Matematiska institutionen, Linköpings universitet, Sweden.

[21] Penrose, R. and Rindler, W. (1984). Spinors and Space-time Vol.1, (Cambridge University Press).

[22] Rainich, G. Y. (1925). Electrodynamics in the general relativity theory, Trans. Amer. Math.Soc., 27, 106-130.

[23] Roberts, M. D. (1988). A new gravitational energy tensor, Gen. Rel. Grav, 20, 775-792.

[24] Robinson, I. (1997). On the Bel-Robinson tensor, C. Q. G., 14, A331-A333.

[25] Senovilla, J. M. M. (2000). Super-energy tensors, C. Q. G., 17, 2799-2841.

[26] Sneddon, G. E. (1996). The identities of the algebraic invariants of the four dimensional Riemann tensor, J. Math. Phys., 37, 1059-1075.

[27] Sneddon, G. E. (1998). The identities of the algebraic invariants of the four-dimensional Riemann tensor. II. $J$. Math. Phys., 39, 1659-1679.

[28] Wheeler, J. A. (1984). Geometrodynamics. Topics of modern physics, vol 1, (Academic Press).

[29] Witten, L. (1959). Geometry of Gravitation and Electromagnetism, Phys. Rev., 115, 206-214.

[30] Zakhary, E. and McIntosh, C. B. G. (1997). A complete set of Riemann invariants, Gen. Rel. Grav, 29, 539-581.

[31] Zund, J. D. (1970). A note on the Bel-Robinson spinor, Tensor, N.S., 21, 354-358. 


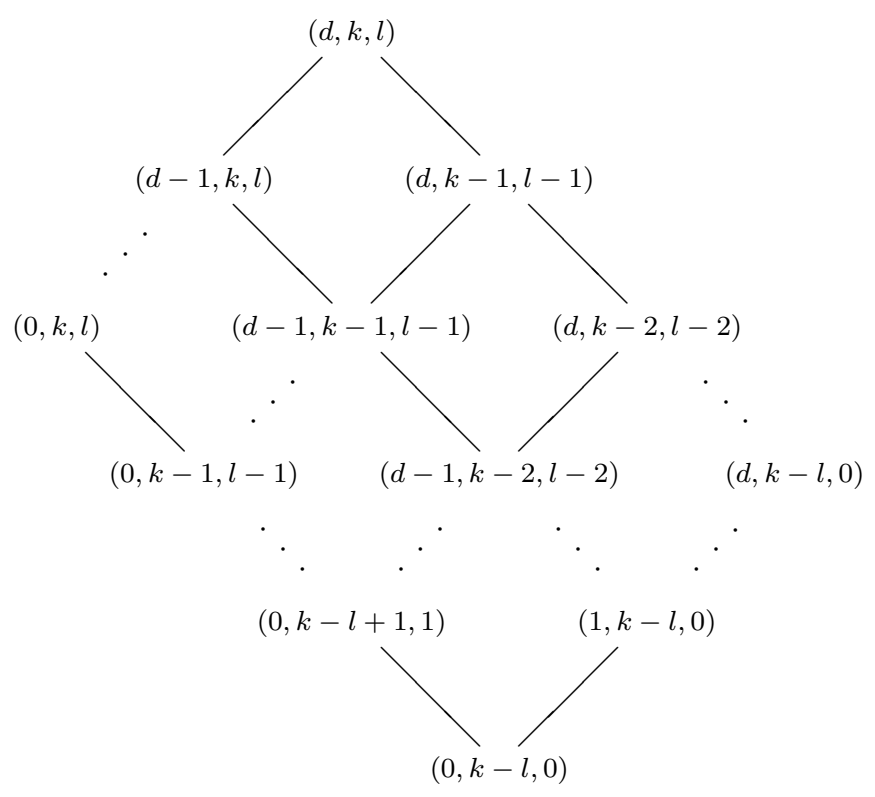

FIG. 1: Sequence of equations

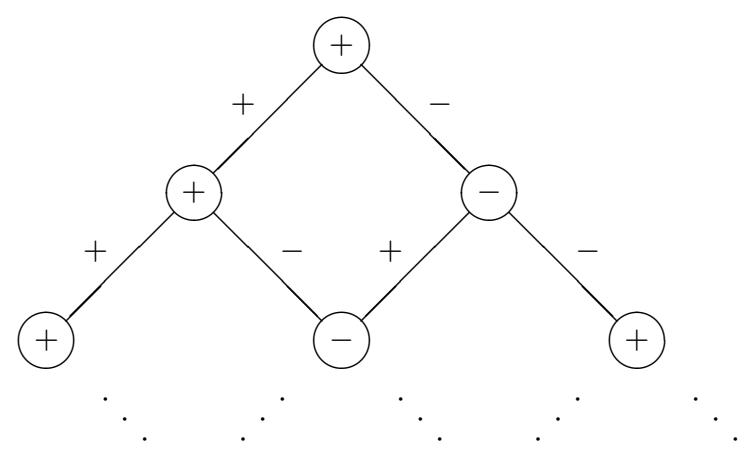

FIG. 2: Signs 